\title{
Design of Radiation Tolerant Electronics for StrECal System in COMET Experiment
}

Kazuki Ueno*, Eitaro Hamada, Masahiro Ikeno, Satoshi Mihara, Hajime Nishiguchi,
Masayoshi Shoji, Tomohisa Uchida KEK

E-mail: kazuuenoepost.kek.ip

Yuki Fujii

Monash University

Ryosuke Kawashima, Kou Oishi, Junji Tojo

Kyushu University

Yu Nakazawa, Hisataka Yoshida

Osaka University

The COMET experiment at J-PARC aims to search for the charged lepton flavor violating process of neutrinoless muon to electron (mu-e) conversion with an improvement of a sensitivity by a factor of 10000 to the current limit. In order to achieve the goal sensitivity, we plan to use a StrECal system as an electron detector, which consists of straw tube tracker (Str) and electron calorimeter (ECal). To read out the signals from the StrECal system precisely, optimal frontend electronics are needed. We have developed the readout electronics boards for Str and ECal called ROESTI and EROS, respectively. Fundamental performance evaluation of ROESTI and EROS was almost done using the prototype boards and it was already found that the performance satisfied the physics requirements. However, those had not satisfied the requirements of radiation tolerance. According to the simulation study, neutron fluence of $10^{12} \mathrm{n} / \mathrm{cm}^{2}$ and gamma-ray absorbed dose of $1 \mathrm{kGy}$ with the safety factor of 5 are expected at the places where the boards are located. Radiation tolerant parts have to be selected and countermeasure against single event upset (SEU) in FPGA has to be considered. Thus, we had parts selection with many times of neutron and gamma-ray irradiation tests. In the results, we found the candidates which satisfied our requirements. Development of new FPGA firmware with the function of SEU detection and correction was also done and its test was done with neutron irradiation. In the result, it was found that the function was effective for the SEU and it satisfied our requirements. Based on those results, we have designed and developed the final version of ROESTI and EROS. In this paper, we describe the details of irradiation tests and those results. The details of the design and performance evaluation of the boards based on the irradiation tests are also reported.

European Physical Society Conference on High Energy Physics - EPS-HEP2019 -

10-17 July, 2019

Ghent, Belgium

${ }^{*}$ Speaker. 


\section{Introduction}

The COherent Muon to Electron Transition (COMET) experiment at J-PARC aims to search for the charged lepton flavor violating process of neutrinoless muon to electron (mu-e) conversion in the presence of a nucleus. This process is strongly restricted by the Standard Model (SM) even if the neutrino oscillation is considered. However, the most of well-motivated theoretical models beyond the SM predict that a branching ratio of this process is to be $\sim 10^{-15}$ [U, [1]. The COMET experiment searches this process at a single-event sensitivity of $\sim 10^{-17}$ which is by a factor of 10000 better than that of the experimental limit given by SINDRUM-II at PSI [3]. When the mu-e conversion occurs, almost all the energy of the muon mass is carried out by the electron which is expected to have the monochromatic energy of about $105 \mathrm{MeV}$. In order to achieve the goal sensitivity, we plan to use a StrECal system as an electron detector, which consists of straw tube tracker (Str) with an excellent momentum resolution and electron calorimeter (ECal) which is used for trigger generation, particle identification and so on. To read out the signals from the StrECal system precisely, optimal front-end electronics are needed. We have developed the readout electronics boards for Str and ECal called ROESTI and EROS, respectively. These boards have the same components except for the front-end part. Fundamental performance evaluation of ROESTI and EROS was almost done using the prototype boards and it was already found that the performance satisfied the physics requirements. However, those had not satisfied the requirements of radiation tolerance. Therefore we have proceeded the radiation studies with simulation and irradiation tests in parallel. Based on the studies, we have designed and developed the final version of ROESTI and EROS.

In this paper, we describe the details of the radiation studies. The details of the design and performance evaluation of the boards based on the irradiation tests are also reported.

\section{Radiation studies}

Simulation studies were done for estimation of the radiation level on detector region by using the PHITS code [田], Geant4 [四], and so on. According to the studies, $1 \mathrm{MeV}$ equivalent neutron fluence of $10^{12} \mathrm{n} / \mathrm{cm}^{2}$ and gamma-ray absorbed dose of $1 \mathrm{kGy}$ with the safety factor of 5 are expected at the places where the boards are located. Radiation tolerant parts have to be selected and countermeasure against single event upset (SEU) in FPGA has to be considered. Thus, we had parts selection, in particular voltage regulator, with many times of neutron and gamma-ray irradiation tests. Many types of switching regulators were tested, and especially LT8612 (Linear Technology) which was one of the candidates was irradiated in some conditions. Neutron irradiation tests were performed at Kobe University Tandem Facility. The neutron is generated by ${ }^{9} \mathrm{Be}(\mathrm{d}, \mathrm{n}){ }^{10} \mathrm{~B}$ reaction, and the energy of the deuteron is $3 \mathrm{MeV} .1 \mathrm{MeV}$ equivalent neutron flux is $4.9 \times 10^{6} \mathrm{n} / \mathrm{cm}^{2} / \mathrm{sec}$ with a beam current of $1 \mu \mathrm{A}$ at $10 \mathrm{~cm}$ from the Be target. In the neutron irradiation test, 4 LT8612s were put on different places with the different distances from the Be target. Gamma-ray irradiation tests were performed at Tokyo Institute of Technology Radiation Research Center. The gamma-ray is provided by the ${ }^{60} \mathrm{Co}$ source, and the dose rate is controlled by changing the distance from the source. In the gamma-ray irradiation test, 3 LT8612s were irradiated with the different dose rate gamma-rays. Fig.W shows the results of irradiation tests of LT8612. In the result, we confirmed that the LT8612 satisfied our requirements..For other components, the candidates were also found. 

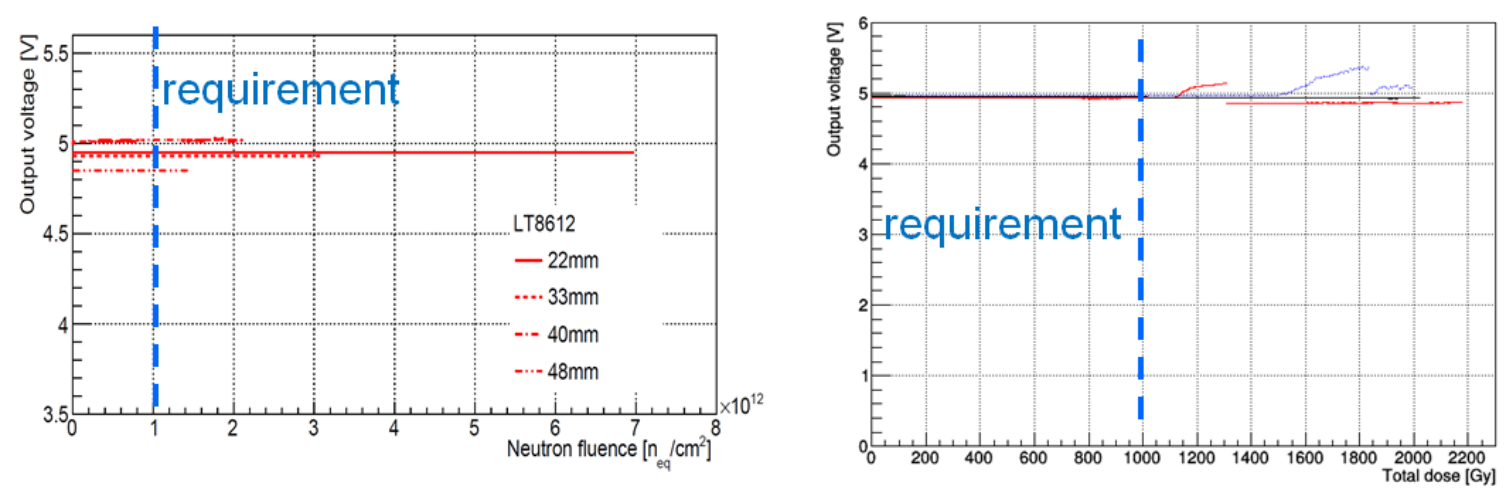

Figure 1: Output voltage of LT8612 as a function of neutron fluence (left) and gamma-ray dose (right). In the neutron irradiation test, 4 LT8612s were put on different places with the different distances from the neutron sources. In the gamma-ray irradiation test, 3 LT8612s were irradiated with the different dose rate gamma-rays.

Development of new FPGA firmware with the function of SEU detection and correction was also done and its test was done with neutron irradiation. In the result, it was found that the function was effective for the SEU and it satisfied our requirements [圆].

\section{Development of StrECal electronics}

Semi-final version of the prototype of the ROESTI was developed and evaluated. As for FPGA, Artix-7(Xilinx) was adopted. As for other parts, radiation tolerant parts were adopted except for regulator, DAC, and SFP which is changed in next version based on the irradiation tests. Fundamental performance evaluation was done and the performance satisfied the requirements from physics $[\square]$. Design of the final version is ongoing.

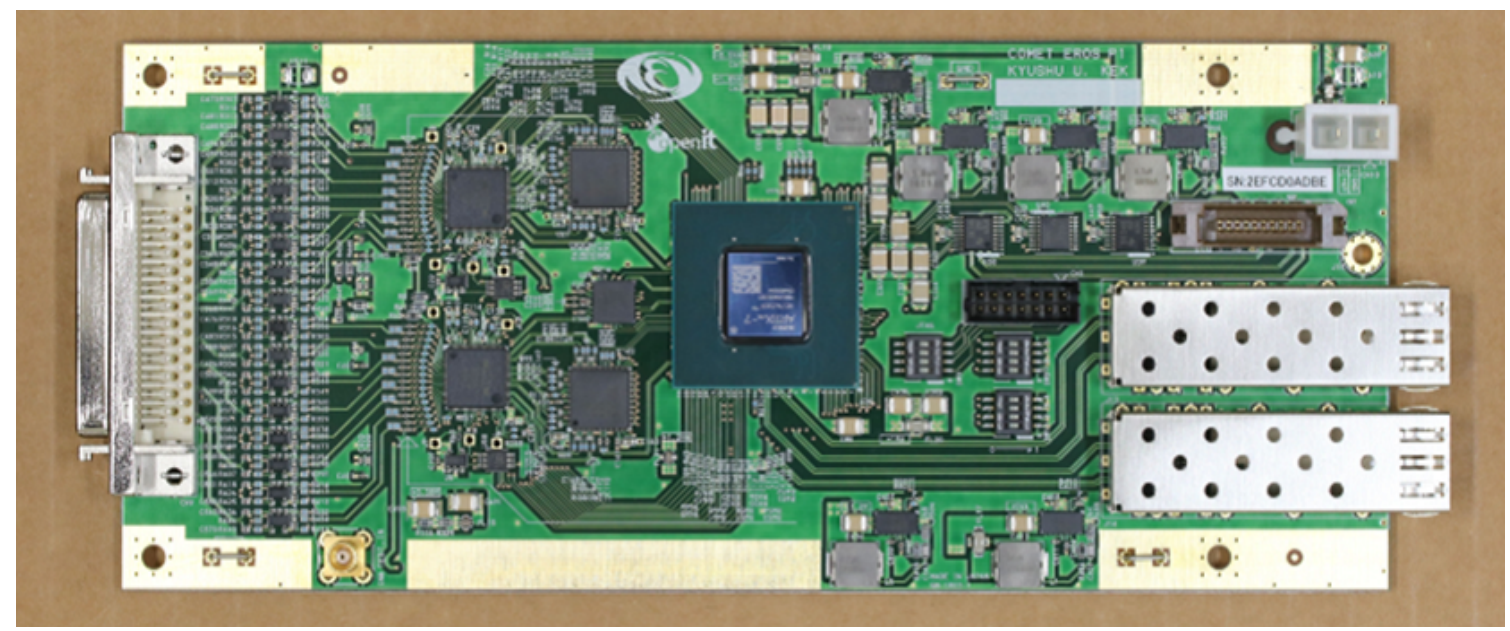

Figure 2: Semi-final version of EROS.

EROS has the same function as ROESTI except for preamplifier. Based on the radiation studies, we developed the semi-final version of the EROS (Fig. 『) in which the LT8612 was adopted. 

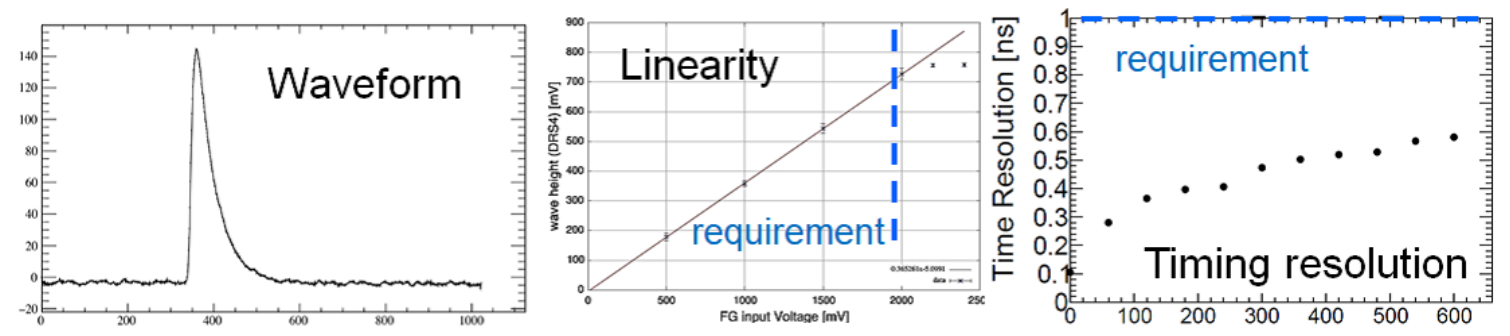

Figure 3: Examples of the fundamental performance of semi-final version of EROS. Example of the waveform from test pulse (left), linearity (center), and timing resolution (right).

Using the semi-final version of the EROS, we evaluated the fundamental performance and confirmed that the performance satisfied our requirements as shown in Fig. B]. Irradiation tests using semi-final version will be done to confirm the radiation tolerance for whole system of the EROS.

\section{Summary}

The COMET experiment aims to search for mu-e conversion process. Radiation tolerant electronics for StrECal system is indispensable. Based on the simulation studies, we have had the many times of the irradiation tests for parts selection, in particular voltage regulator. In the results of the neutron and gamma-ray irradiation tests, we confirmed that LT8612 satisfied our requirements. Using the regulator, the semi-final version of the electronics board was developed and evaluated, then it was confirmed that the fundamental performance satisfied our requirements. We will have irradiation test of the whole system of the semi-final version in near future. After the test, we will construce the final version of the EROS and ROESTI.

\section{Acknowledgments}

The development of readout electronics is supported by Open-It. This research is supported by the grant-in-aids, JSPS KAKENHI 17H06135 and 17H04841.

\section{References}

[1] Y. Kuno and Y. Okada, Rev. Mod. Phys. 73 (2001) 151.

[2] R. Kitano, M. Koike, S. Komine, Y. Okada, Phys. Lett. B 575 (2003) 300.

[3] W. Bertl, et al. Eur. Phys. J. C 47 (2006) 337.

[4] T. Sato, et al., J. Nucl. Sci. Technol. 50:9 (2013) 913.

[5] S. Agostineli et al., Nucl. Instrum. Methods A 506 (2003) 250

[6] Y. Nakazawa, et al., Nucl. Instrum. Methods A 936 (2019) 351.

[7] K. Ueno, et al., Nucl. Instrum. Methods A 936 (2019) 297. 